Check for updates

Cite this: Chem. Sci., 2019, 10, 3854

๑ All publication charges for this article have been paid for by the Royal Society of Chemistry

Received 23rd January 2019

Accepted 21st February 2019

DOI: $10.1039 / \mathrm{c} 9 \mathrm{sc} 00384 \mathrm{c}$

rsc.li/chemical-science

\section{Davydov splitting and singlet fission in excitonically coupled pentacene dimerstt}

Bettina Sabine Basel, $\S^{a}$ Constantin Hetzer, $\S^{\mathrm{b}}$ Johannes Zirzlmeier, $\S^{\mathrm{a}}$ Dominik Thiel, ${ }^{a}$ Rebecca Guldi, ${ }^{\mathrm{b}}$ Frank Hampel, (D) ${ }^{\mathrm{b}}$ Axel Kahnt, (D) ${ }^{\mathrm{c}}$ Timothy Clark, (D) *d Dirk Michael Guldi (D) ${ }^{* a}$ and Rik R. Tykwinski (D)

Singlet fission (SF) allows two charges to be generated from the absorption of a single photon and is, therefore, potentially transformative toward improving solar energy conversion. Key to the present study of SF is the design of pentacene dimers featuring a xanthene linker that strictly places two pentacene chromophores in a rigid arrangement and, in turn, enforces efficient, intramolecular $\pi$-overlap that mimics interactions typically found in condensed state (e.g., solids, films, etc.). Inter-chromophore communication ensures Davydov splitting, which plays an unprecedented role toward achieving SF in pentacene dimers. Transient absorption measurements document that intramolecular SF evolves upon excitation into the lower Davydov bands to form a correlated triplet pair at cryogenic temperature. At room temperature, the two spin-correlated triplets, one per pentacene moiety within the dimers, are electronically coupled to an excimer state. The presented results are transferable to a broad range of acene morphologies including aggregates, crystals, and films.

\section{Introduction}

Singlet fission (SF) is a spin-allowed photophysical process, in which an excited singlet state is transformed rapidly and efficiently into two triplet excited states. ${ }^{1-5}$ Although SF was first observed more than fifty years ago in crystalline systems, ${ }^{6-8}$ interest has only recently been rekindled, as SF could serve as a means to overcome the theoretical limit for solar cell performance first introduced by Shockley and Queisser. ${ }^{9-14}$ Chromophores must fulfill a basic requirement of energy conservation

${ }^{a}$ Department of Chemistry and Pharmacy, Interdisciplinary Center for Molecular Materials (ICMM), Friedrich-Alexander-Universität (FAU), Egerlandstrasse 3, 91058 Erlangen, Germany. E-mail: dirk.guldi@fau.de

${ }^{b}$ Department of Chemistry and Pharmacy, Interdisciplinary Center for Molecular Materials (ICMM), Friedrich-Alexander-Universität (FAU), Nikolaus-Fiebiger-Strasse 10, 91058 Erlangen, Germany

${ }^{c}$ Leibniz Institute of Surface Engineering (IOM), Permoserstr. 15, D-04318 Leipzig, Germany

${ }^{d}$ Department of Chemistry and Pharmacy, Computer-Chemistry-Center (CCC), Friedrich-Alexander-Universität Erlangen-Nürnberg (FAU), Nägelsbachstrasse 25, 91052 Erlangen, Germany. E-mail: tim.clark@fau.de

${ }^{e}$ Department of Chemistry, University of Alberta, Edmonton, Alberta, T6G 2G2, Canada.E-mail: rik.tykwinski@ualberta.ca

$\dagger$ Dedicated to Professor Fritz Wasgestian on the occasion of his 85th birthday. \$ Electronic supplementary information (ESI) available: Supplemental Information includes synthetic protocols, details about the analysis of photophysical data, 50 figures and X-ray crystallographic data for 1a (cif), 1d (cif), 4b (cif), and 4c (cif). CCDC 1839936, 1839935, 1839934 and 1839933. For ESI and crystallographic data in CIF or other electronic format see DOI: $10.1039 / \mathrm{c} 9 \mathrm{sc} 00384 \mathrm{c}$

$\S$ These authors contributed equally. for SF, in which the first singlet excited-state energy must be similar to or greater than twice the energy of the lowest-lying triplet excited state; $E\left(\mathrm{~S}_{1}\right) \approx 2 E\left(\mathrm{~T}_{1}\right)$ or $E\left(\mathrm{~S}_{1}\right)>2 E\left(\mathrm{~T}_{1}\right){ }^{1-5,15,16}$ In addition, rates play a crucial role in determining the efficiency of SF; the chromophore-system should demonstrate fast conversion of the singlet locally excited ${ }^{1}\left(\mathrm{~S}_{1} \mathrm{~S}_{0}\right)$ state into two spin-coupled local triplet excited ${ }^{1}\left(T_{1} T_{1}\right)$ states in order to compete efficiently with parasitic deactivation pathways such as fluorescence, internal conversion, and intersystem crossing..$^{1-5}$ The rate of SF depends strongly on the electronic coupling matrix elements between the chromophores and the free-energy difference between the states involved., ${ }^{4,5}$

Substantial efforts have been made to develop molecular systems suitable for efficient SF, and the number of chromophores that fulfill the necessary requirements, although somewhat limited, is currently increasing to include acenes, ${ }^{17-22}$ diphenylisobenzofurans, ${ }^{23-25}$ carotenoids, ${ }^{26,27}$ rylenes, ${ }^{28-30}$ and others. ${ }^{31-33}$ With a wider selection of potential SF-chromophores available, the more difficult task of deciphering the mechanistic and morphological details associated with SF must now be addressed.

Three different SF mechanisms have been proposed so far: the direct mechanism, ${ }^{34,35}$ where the $\left(\mathrm{S}_{1} \mathrm{~S}_{0}\right)$ state decays directly to ${ }^{1}\left(T_{1} T_{1}\right)$; the mediated mechanism, in which the ${ }^{1}\left(T_{1} T_{1}\right)$ formation is facilitated by coupling to a (virtual) charge-transfer (CT) intermediate; ${ }^{36,37}$ and the state-mixing or quantum coherent mechanism, where a coherent superposition of the $\left(\mathrm{S}_{1} \mathrm{~S}_{0}\right),{ }^{1}\left(\mathrm{~T}_{1} \mathrm{~T}_{1}\right)$ and (sometimes) the CT state, is generated directly after excitation. $^{38,39}$ 
Studies of SF in the solid state are often challenging because of timescales that are too fast to allow mechanistic aspects to be unraveled. Moreover, independent fine-tuning of mechanistic variables such as polarity, inter-chromophore distance, and the effective $\pi$-conjugation between two chromophores can be very complex in solid-state samples. To overcome the limitations of solid-state analyses, SF in specially designed dimers has been investigated in dilute solution. Under such conditions, the rate of SF is reduced, and valuable insight regarding factors that influence the mechanism and yield can be elucidated more easily. ${ }^{20,36,40-45}$ An important difference between measurements in solution and in the solid state arises from the significant electronic influence of neighboring molecules in the latter. Packing of pentacene derivatives in the solid state, for example, is often characterized by significant, direct $\pi$-overlap of neighboring chromophores. ${ }^{46-49}$

Strong intermolecular $\pi-\pi$ interactions, then, lead to interchromophore coupling and Davydov splitting. More specifically, Davydov splitting arises from the overlap of the wavefunctions of two translationally inequivalent molecules in the unit cells of crystals. ${ }^{46,50}$ To be consistent with previous reports on acene dimers, ${ }^{51-54}$ we refer to Davydov splitting rather than the synonymous exciton splitting. ${ }^{55}$ Excited-state properties and dynamics that lead to Davydov splitting have been reported for e.g., polycrystalline single crystals,${ }^{50}$ acene films, ${ }^{56}$ and nanoaggregates. ${ }^{57,58}$ Davydov splitting of the lowest singlet excited state has been predicted for pentacene crystals to lead to an electronic situation that is favorable for fast SF. ${ }^{59}$ Importantly, it has been shown for crystalline perfluoropentacene that excimer states formed from Davydov states act as intermediates that favor SF, rather than compete with it. ${ }^{60}$ For films of diketopyrrolopyrroles with marked Davydov splitting, excimer states formed on photoexcitation have been identified as a multiexcitonic ${ }^{1}\left(\mathrm{~T}_{1} \mathrm{~T}_{1}\right)$ state en route to triplet decorrelation. ${ }^{61}$

In the case of dimers containing two "monomeric" chromophores, overlap of the wavefunction localized on each of the two monomers leads to Davydov splitting into two dimer states. ${ }^{51}$ In dilute solutions, direct $\pi$-overlap in, for example, perylenediimide dimers leads to Davydov splitting, which results in a scenario in which excimer formation is favored and $\mathrm{SF}$ is disfavored. ${ }^{62,63}$ Lately, a lively debate has arisen about the nature of excimer states in tetracenes and pentacenes. In the case of concentrated TIPS-tetracene solutions, a correlated triplet pair state with excimer character was identified, ${ }^{\mathbf{6}}$ whereas in tetracene dimers a $\mathrm{S}_{1^{-}}$ excimer state acts as intermediate in SF. ${ }^{19}$ The impact of a CTcharacter is also highly controversial. No CT-character was found for singlet excimer states, which mediate SF in microcrystalline pentacene films with Davydov splitting of the $S_{1} \leftarrow S_{0}$ transition. ${ }^{65}$ In contrast, singlet excited states in pentacene single crystals, which show Davydov splitting of the $S_{1}$ state, are predicted to possess CT-character and support SF. ${ }^{59}$ Different conclusions are also drawn for the role of acene-centered excimer states in SF. On the one hand, excimers states in concentrated TIPS-tetracene solutions with absorption features of singlet and triplet states are identified as intermediates in SF. ${ }^{64}$ On the other hand, excimer states found in the same system, are diagnosed to act as trap states that are detrimental to SF. ${ }^{66}$ These observations are consistent with the fact that a competition exists in tetracene dimers between excimer formation and SF. ${ }^{\mathbf{5 4 , 6 7}}$

Such debates underline the necessity to test the role and nature of excimer states in acene-based SF. We opted for Davydov splitting as a suitable parameter to distinguish between different kinds of excimers and their corresponding roles. Our idea is based on the nature of Davydov splitting: it is caused by electronic interactions between, for example, neighboring acenes in their electronic ground-states. This contrasts excimer states, which emerge when two neighboring acenes interact solely in their electronically excited state. ${ }^{65}$ The focus of our investigation is to dissect the role of excimers, which emerge from photoexcitable dimer states in the form of Davydov splitting. Notably, SF in pentacene dimers that show Davydov splitting in solution has not been demonstrated to date.

In the present study, we have designed four pentacene dimers, in which a xanthene linker is used to position the two pentacene-chromophores in a rigid arrangement, which, in turn, enforces strong co-facial $\pi-\pi$-interactions. $\Upsilon^{20,68}$ Using these pentacene dimers in dilute solution, we outline the role of inter-chromophore coupling through direct $\pi$-overlap, which leads to solid-state-like Davydov splitting. Transient absorption spectroscopy enables us to define the influence of Davydov splitting on the excited-state processes. In particular, we outline excimer state formation following excitation of Davydov split pentacene energy levels in dilute solution and at variable temperatures. In doing so, we confirm fast SF and identify the nature of excimer states in excitonically coupled pentacenes.

\section{Results and discussion}

\section{Synthesis}

The synthesis of xanthene-linked pentacene dimers was based on the Sonogashira cross-coupling of terminal alkyne building blocks 1a-1d ${ }^{69}$ with 4,5-diiodo-2,7-di-tert-butyl-9,9-dimethylxanthene $\mathbf{2}^{\mathbf{7 0}}$ to give intermediates 3a-c in 35-62\% yield, respectively (Scheme 1, see ESIt for synthetic details). In the case of 3d, the desired product could not be isolated pure, and, therefore, the crude mixture was taken directly on to the next step without further purification. With $\mathbf{3 a - d}$ in hand, $\mathrm{SnCl}_{2}$-mediated reductive aromatization $^{71}$ in the presence of $\mathrm{H}_{2} \mathrm{SO}_{4}$ furnished dimers 4ad in good yields. Compounds $\mathbf{4 a - d}$ are stable toward air and moisture under normal laboratory conditions and show good solubility in common organic solvents like $\mathrm{CHCl}_{3}, \mathrm{CH}_{2} \mathrm{Cl}_{2}, \mathrm{THF}$, and toluene ( $c a .6 \mathrm{mg} \mathrm{mL} \mathrm{m}^{-1}$ ). Thermal analysis of $4 \mathbf{4 a}-\mathbf{d}$ via differential scanning calorimetry revealed decomposition temperatures that range from $240{ }^{\circ} \mathrm{C}$ for $\mathbf{4 d}$ to $296^{\circ} \mathbf{C}$ for $\mathbf{4 b}$.

\section{Structural analysis}

Crystallographic analyses have established that acene dimers can adopt either a "parallel" or "twisted" orientation of the two chromophores in the solid state. ${ }^{19} \mathrm{X}$-ray analysis of dimers $\mathbf{4 b}$ and $4 \mathbf{c}$ reveals a parallel alignment of the two pentacene moieties - Fig. S22-S25. The structures of both dimers highlight the intramolecular proximity of the two pentacenemoieties with close contacts in the solid of 3.3-3.6 $\AA$ for $4 \mathbf{b}$ 

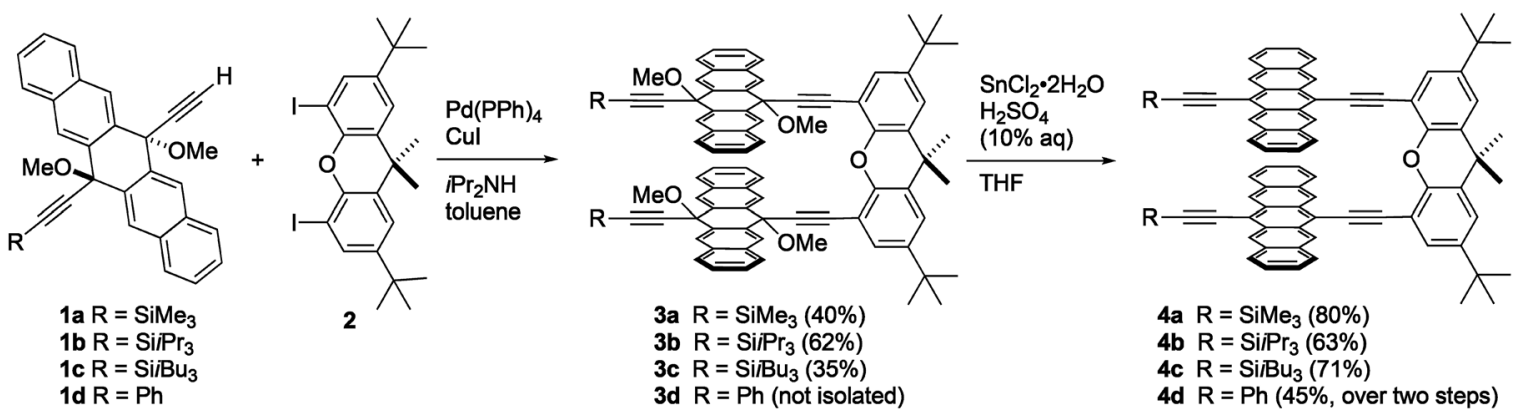

Scheme 1 Synthesis of xanthene-linked dimers $4 a-d$.

and 3.0-3.5 $\AA$ for $4 \mathbf{c}$. Importantly, the increased steric bulk of the more solubilizing $\mathrm{Si} \mathrm{Bu}_{3}$ does not appear to significantly affect the stacking distances between pentacenes in the solid state, although the relative intramolecular orientation of the two pentacenes differs slightly - Fig. S23.ł

\section{Calculations - insight into Davydov splitting}

Two lowest energy conformations were found for $\mathbf{4 a - d}$ by classical molecular-dynamics (MD) simulations in the gas phase (Fig. 1). The two conformations identified in the MD simulations were optimized using density-functional theory (DFT) with the B3LYP65 and $\omega$ B97XD66 density functionals, the former were augmented by the D3 dispersion corrections. ${ }^{72-75}$ All DFTcalculations used the 6-31G(d) basis set. Initial calculations for 4a showed that the xanthene substitution (i.e., $\mathrm{X}=t \mathrm{Bu}$ in Fig. 1) does not affect the relative stabilities of the "parallel" and "twisted" conformations, so that the remaining calculations were performed on the unsubstituted derivatives $\mathbf{4} \mathbf{a}^{\prime}-\mathbf{4} \mathbf{d}^{\prime}$ (i.e., $\left.\mathrm{X}=\mathrm{H}\right)$.

Table 1 shows the results of the DFT calculations, which emphasize that, in the absence of intermolecular crystalpacking interactions, the twisted conformation is more stable in each dimer. The two density functionals agree quite closely on the relative stabilities of the two conformations. The different conformational preference between solution and solid-state structures is attributed to intermolecular interactions in the latter. The "parallel" conformer is found to be between 4 and $20 \mathrm{kcal} \mathrm{mol}^{-1}$ less stable than the "twisted" conformer - Table 1 . For both $\mathbf{4} \mathbf{b}^{\prime}$ and $\mathbf{4} \mathbf{c}^{\prime}$, the conformations of the substituents may, however, not be the most stable one, so that the relative energies for these compounds must be treated
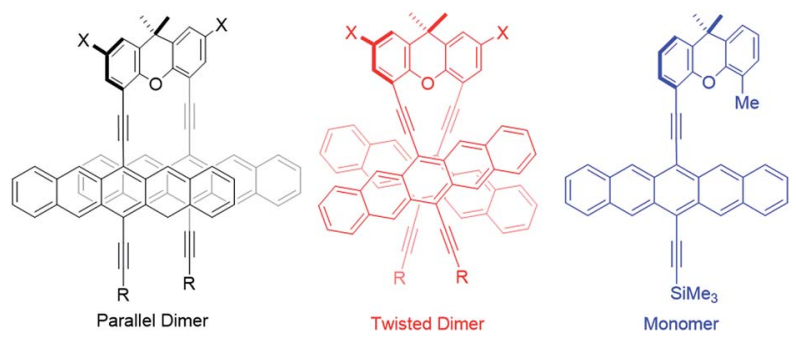

Fig. 1 Schematic views of the parallel and twisted conformations as well as the monomer used as a reference for energy-level calculation. $4 \mathrm{a}\left(\mathrm{R}=\mathrm{Me}_{3} \mathrm{Si} ; \mathrm{X}=t \mathrm{Bu}\right)$ and $4 \mathrm{a}^{\prime}-\mathrm{d}^{\prime}\left(\mathrm{R}=\mathrm{Me}_{3} \mathrm{Si}, \mathrm{Pr}_{3} \mathrm{Si}, i \mathrm{Bu} \mathrm{u}_{3} \mathrm{Si}, \mathrm{Ph} ; \mathrm{X}=\mathrm{H}\right)$. with caution. Nonetheless, we can conclude from the DFT calculations that the twisted conformation should predominate in solution. As is evident from Fig. 1, the $\pi$-overlap between the pentacene moieties is larger in the parallel than in the "twisted" conformation. Please note that this does not have a large effect on the calculated spectra. A comparison of the absorption spectra of the two conformers using the semiempirical AM1 Hamiltonian $^{76}$ with configuration interaction including only single excitations (CIS) and an active space of 64 occupied and 64 virtual orbitals and polarized-continuum model solvation in benzonitrile is given in Fig. S26.\$

Exciton/Davydov splitting is often discussed within the context of $\mathrm{H}-/ \mathrm{J}$-coupling: ${ }^{77}$ the upper Davydov band relates here to the $\mathrm{H}$-feature, while the lower is synonymous with the J-feature. ${ }^{.8}$ To discuss the Davydov splitting in $\mathbf{4 a - d}$ in terms of $\mathrm{H}$ - and J-bands, however, would be at least partially incorrect as $\mathrm{H}-\mathrm{J}$-couplings are only applicable for coplanar and parallel configurations. ${ }^{55,77}$ The twisted conformers of $4 a-d$ that predominate in solution do not conform to the coplanar/ parallel picture. Therefore, we believe that the terms upper and lower Davydov bands provide a more accurate description.

Calculations show that Davydov splitting is observed for all singlet excited states, albeit small $(<0.03 \mathrm{eV})$ except for the lowest singlet excited state, which is split by $0.35 \mathrm{eV}$ in the twisted conformer of 4a (Fig. 2). The corresponding splitting in the "parallel" conformation is lower $(0.26 \mathrm{eV})$. In contrast, appreciable Davydov splitting is predicted to be absent in a corresponding monomer that features only one pentacene moiety. On the other hand, both the calculations and experiments suggest that, while Davydov splitting undoubtedly dominates our observations, other considerations likely complicate the spectrum. The Davydov-split bands (combinations of HOMO $\rightarrow$ LUMO and HOMO-1 $\rightarrow$ LUMO+1), the lower-energy of which is calculated to have very low intensity (calculated oscillator strength 0.031), mix with other single excitations, so that the splitting is larger than expected. More specifically, the lower band mixes with HOMO $\rightarrow$ LUMO+1 and HOMO-1 $\rightarrow$ LUMO, and the higher one with HOMO-5 $\rightarrow$ LUMO+6 and HOMO-6 $\rightarrow$ LUMO+5 (relevant orbitals are shown in Fig. S28:). Most importantly, these orbitals are unsymmetrical combinations of $\pi$-MOs centered on the two chromophores and, in turn, rather strong mixing with the Davydov bands is possible. When turning to the triplet excited state, a much smaller splitting of $0.007 \mathrm{eV}$ is found. 
Table 1 DFT-calculated energies for the pentacene dimers ${ }^{a}$

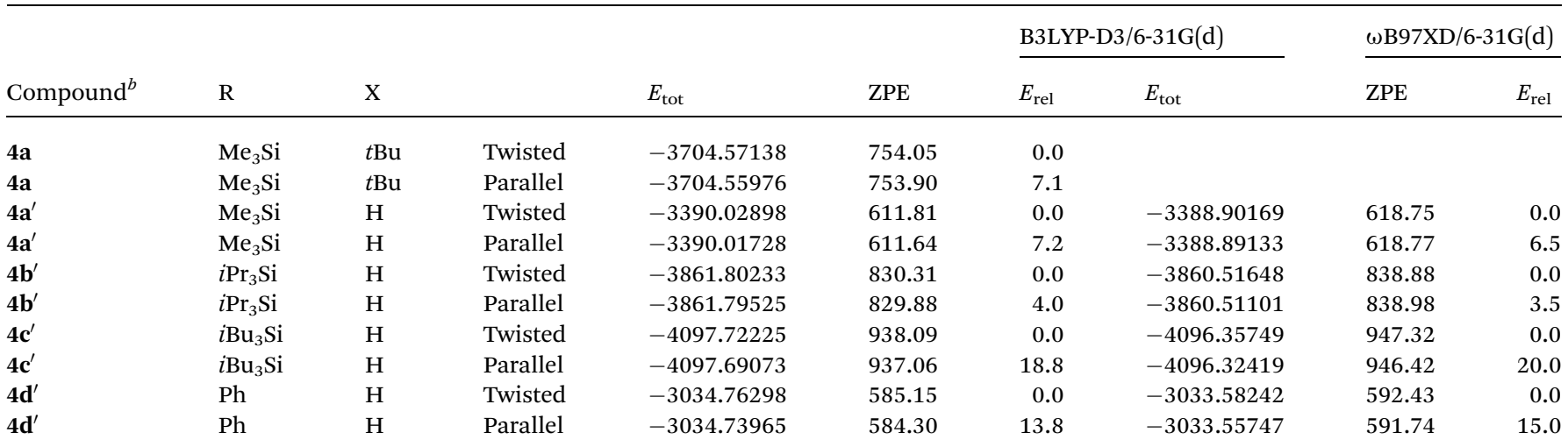

${ }^{a} E_{\text {tot }}[$ a.u. $]=$ total energies, ZPE $\left[\mathrm{kcal} \mathrm{mol}^{-1}\right]=$ zero-point vibrational energies, $E_{\text {rel }}\left[\mathrm{kcal} \mathrm{mol}^{-1}\right]=$ relative energies $\left(E_{\text {rel }}=\right.$ Born-Oppenheimer + ZPE). ${ }^{b}$ See Fig. 1 for molecular structures.

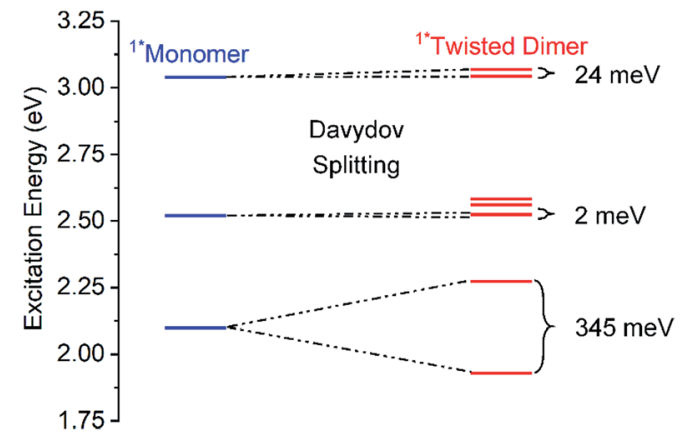

Fig. 2 Energy-level diagram of the calculated singlet excited states of the twisted dimer conformation of $4 a$ (red) in comparison to the monomer reference (blue). Davydov splitting is indicated by the correlation lines and the splitting energy is given in meV. For the corresponding triplet excited state levels see Fig. S27.

\section{Steady state absorption and electrochemistry - observation of Davydov splitting}

To confirm intramolecular Davydov splitting, we performed steady state absorption measurements with $\mathbf{4 a - d}$ at room temperature in toluene, THF, and benzonitrile; 6,13-bis(triisobutylsilylethynyl)pentacene (TIBS) was used as a reference. A comparison of the spectra for $\mathbf{4 a - d}$ and TIBS reveals a number of distinct differences. First, all vibronic fine structure in the absorption spectra of $\mathbf{4 a - d}$ show a red shift of about $20 \mathrm{~nm}$ compared to TIBS, as a result of stabilization by van der Waals interactions. ${ }^{77}$ Second, in all solvents, the extinction coefficients across the visible range are lower than the linear sum of two pentacene moieties. This effect is particularly strong for the fundamental $0-0 *$ absorption at around $660 \mathrm{~nm}$ - Fig. 3. Third, additional absorption features develop in the long-wavelength region between 680 and $800 \mathrm{~nm}$ (Fig. 3).

To rule out that these results stem from aggregation, their concentration dependency was examined, and it was established that the Lambert-Beer relationship was upheld - Fig. 4 and S30S34. In short, the appearance of the additional absorptions is consistent with Davydov splitting. The Davydov features show solvent dependency, and they are poorly resolved in THF, slightly
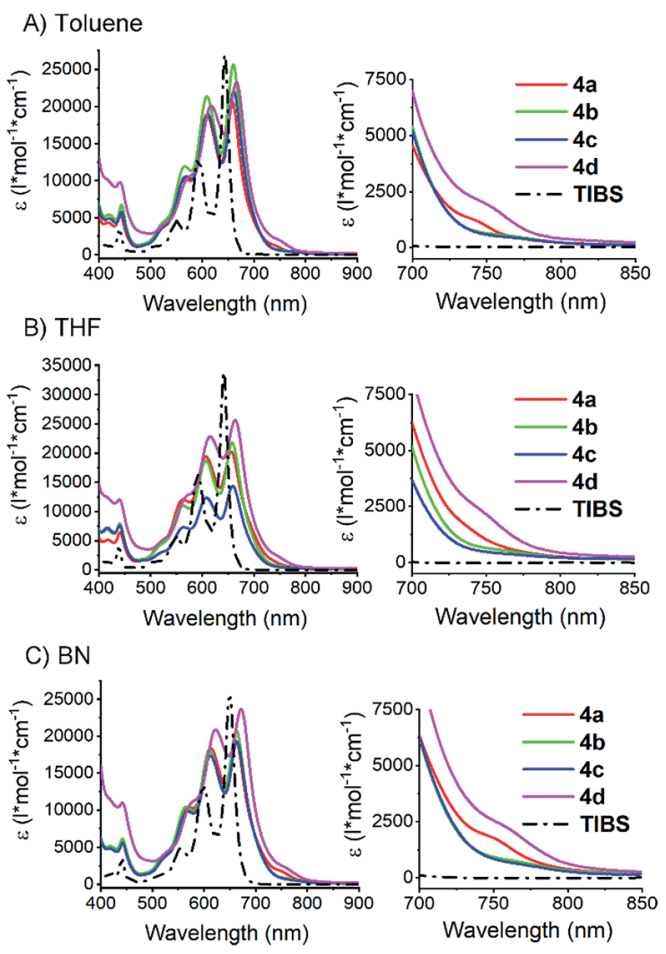

Fig. 3 Steady state absorption spectra of dimers $4 a-d$ and TIBS in different solvents. Measured in (A) toluene, (B) THF, and (C) benzonitrile $(B N)$ at room temperature. On the right: expansions of the absorption features that are identified as lower energy Davydov bands (see also Fig. S29\$).

better resolved in toluene, and clearly visible in benzonitrile Fig. 3 (especially for compounds $\mathbf{4 a}$ and $4 \mathbf{d}$ ). Specifically, the solvent viscosity increases from THF $\left(\eta\left(25^{\circ} \mathrm{C}\right)=0.456 \mathrm{mPa}\right)$ to toluene $\left(\eta\left(25^{\circ} \mathrm{C}\right)=0.560 \mathrm{mPa} \mathrm{s}\right)$ and to benzonitrile $\left(\eta\left(25^{\circ} \mathrm{C}\right)=\right.$ $1.267 \mathrm{mPa} \mathrm{s}),{ }^{79}$ suggesting Davydov splitting is contingent on the solvent viscosity. Considering the impact that viscosity exerts on nuclear motions, our findings are rationalized in terms of cooling of intramolecular vibrations and, in turn, freezing intramolecular $\pi$-overlap between the two pentacenes. Independent confirmation for this hypothesis comes from 
A) $4 \mathbf{a}$ in $\mathrm{BN}$

C) $4 \mathrm{c}$ in $\mathrm{BN}$

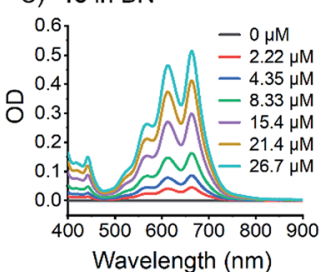

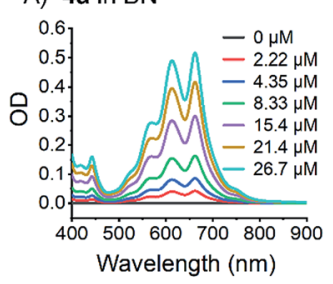

B) $4 \mathrm{~b}$ in $\mathrm{BN}$

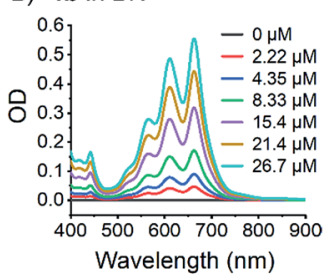

D) $4 \mathrm{~d}$ in $\mathrm{BN}$

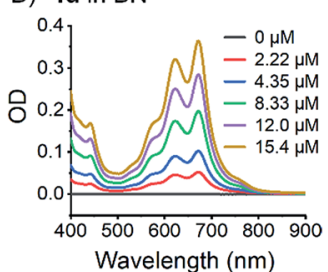

Fig. 4 Steady state absorption spectra of dimers $4 a-d(A)-(D)$ in benzonitrile $(\mathrm{BN})$ at different concentrations and room temperature.

temperature-dependent absorption measurements in 2-methyltetrahydrofuran (MeTHF) - Fig. 5. In particular, the Davydov bands, which were poorly resolved at room temperature, are seen as distinct maxima in the lower temperature regime.

Davydov splitting in $\mathbf{4 a - d}$ is also supported by electrochemical characterization. Rather than observing a single, twoelectron oxidation at around $+0.45 \mathrm{~V}$ as seen in the case of the one-electron oxidation of TIBS, a pair of oxidations is observed: +0.22 and $+0.51 \mathrm{~V}$ for $4 \mathrm{a},+0.19$ and $+0.50 \mathrm{~V}$ for $4 \mathbf{b}$, and +0.20 and $+0.51 \mathrm{~V}$ for $4 \mathrm{c}-$ Fig. S35. $\$$ All values are given versus $\mathrm{Fc} / \mathrm{Fc}^{+}$ (ferrocene/ferrocenium). $\|$ At the same time, a single twoelectron reduction is noted for $\mathbf{4 a}-\mathbf{c}$ at $c a$. $-1.6 \mathrm{~V}$, which is slightly more negative than seen for TIBS at $-1.44 \mathrm{~V}$. As a matter of fact, the two different oxidations agree with AM1 full CI calculations. Here, we considered two occupied and two virtual orbitals in the active space as well as an SCRF solvent model for benzonitrile. The splitting is $0.2 \mathrm{eV}$ between the two lowest-lying doublet states of the molecular radical cation at the $\omega$ B97XDoptimized geometry of the neutral molecule.
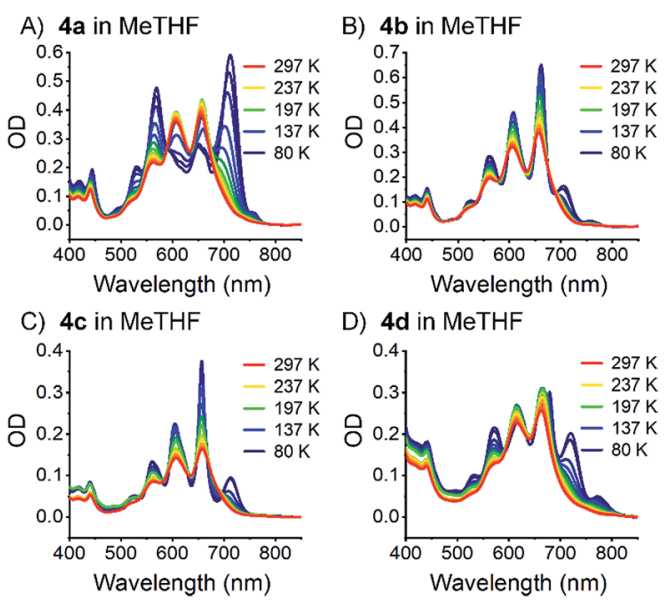

D) $\mathbf{4 d}$ in MeTHF

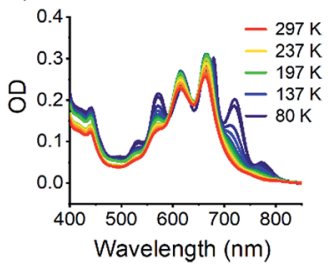

Fig. 5 Steady state absorption spectra of dimers $4 a-d(A)-(D)$ measured in MeTHF at variable temperatures (80 to $297 \mathrm{~K}$ ).
Transient absorption measurements at cryogenic temperatures - observing singlet fission

To observe the excited-state dynamics upon exciting Davydov levels, femtosecond transient absorption (fsTA) spectroscopy was performed at $80 \mathrm{~K}$ by irradiation at $730 \mathrm{~nm} .730 \mathrm{~nm}$ excites the dimers into their low-energy Davydov bands. The datasets obtained were analyzed with multi-wavelength and global analyses (see ESI† for details). A global analysis with a sequential model was performed to identify the decay dynamics. Low temperature experiments were performed in MeTHF.

Upon photoexcitation, the typical fingerprint absorptions of the pentacene singlet excited $\left(\mathrm{S}_{1} \mathrm{~S}_{0}\right)$ state at around $1400 \mathrm{~nm}$ form instantaneously for all four dimers ${ }^{36}$ - Fig. 6. ${ }^{* *}$ The $\left(S_{1} S_{0}\right)$ state decays with lifetimes of less than 200 fs and new absorption features, which evolve at around 480 and $515 \mathrm{~nm}$, are in sound agreement with the pentacene triplet excited state (Fig. 7 and S36). Independent evidence for our spectral assignment comes from triplet sensitization experiments with $N$-methylfulleropyrrolidine ( $N$-MFP) - Fig. S40.\$ $\$^{80}$ This result, in combination with the very fast formation dynamics ( $<200 \mathrm{fs})$, substantiates the notion of intramolecular SF. In turn, the triplet features indicate the presence of the $\left(\mathrm{T}_{1} \mathrm{~T}_{1}\right)$ state.

Interestingly, the decay of the $\left(\mathrm{T}_{1} \mathrm{~T}_{1}\right)$ state is biphasic for $\mathbf{4 b}$, 4c, and 4d. Consequently, we used a kinetic model with two states, $\left(T_{1} T_{1}\right)_{\tau 1}$ and $\left(T_{1} T_{1}\right)_{\tau 2}$, for the global fit. We considered two different options, parallel (Fig. S38\%) and sequential (Fig. 7) models. In the former, $\left(\mathrm{T}_{1} \mathrm{~T}_{1}\right)_{\tau 1}$ and $\left(\mathrm{T}_{1} \mathrm{~T}_{1}\right)_{\tau 2}$ are populated simultaneously and decay in parallel. In the latter, $\left(T_{1} T_{1}\right)_{\tau 1}$ is populated initially and decays to $\left(T_{1} T_{1}\right)_{\tau 2}$. Importantly, the $\left(\mathrm{T}_{1} \mathrm{~T}_{1}\right)_{\tau 1}$ spectrum agrees much better with the triplet spectra generated by photosensitization with $N$-MFP for the sequential rather than parallel model. Much more of
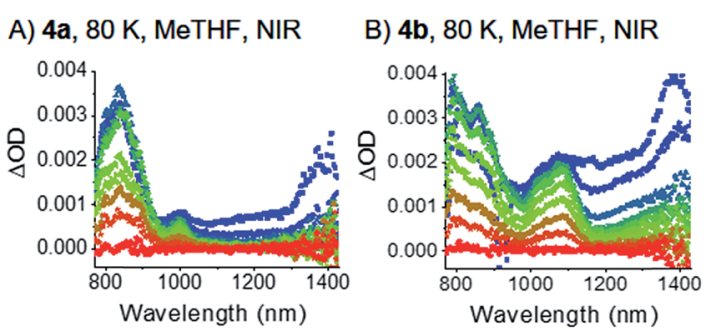

C) $4 \mathrm{c}, 80 \mathrm{~K}, \mathrm{MeTHF}, \mathrm{NIR}$
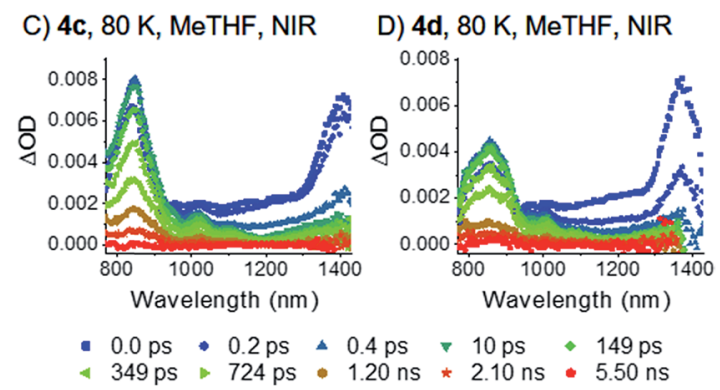

Fig. 6 Transient optical absorption spectra of dimers $4 a-d(A)-(D)$ in frozen argon-saturated MeTHF. The data were obtained upon femtosecond pump-probe experiments with an excitation of $730 \mathrm{~nm}$ and detection in the near-infrared (NIR) at $80 \mathrm{~K}$. The raw data were chirp, zero point, and baseline corrected. 
A) EAS, $80 \mathrm{~K}, \mathrm{MeTHF}, 4 \mathrm{a}$

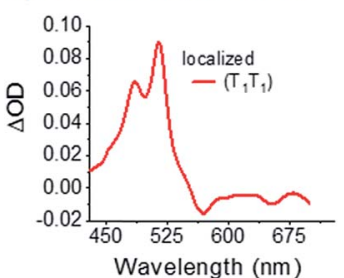

C) EAS, $80 \mathrm{~K}, \mathrm{MeTHF}, 4 \mathrm{c}$

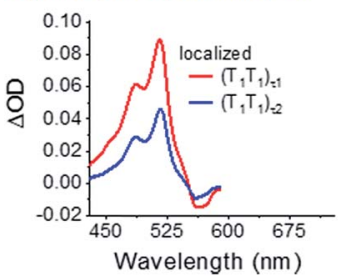

B) EAS, $80 \mathrm{~K}, \mathrm{MeTHF}, 4 \mathrm{~b}$

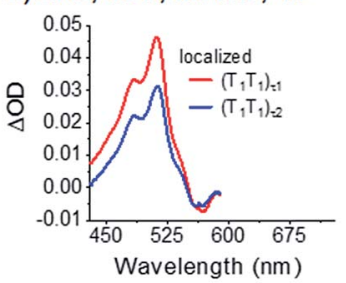

D) EAS, $80 \mathrm{~K}, \mathrm{MeTHF}, 4 \mathrm{~d}$

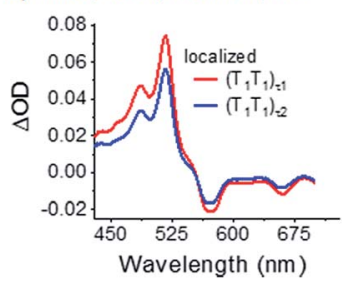

Fig. 7 EAS of dimers $4 a-d(A)-(D)$ in frozen argon-saturated MeTHF, VIS. The data were recorded at $80 \mathrm{~K}$. Spectra were obtained by global analysis with a sequential model of the transient absorption data measured upon femtosecond pump-probe experiments with $730 \mathrm{~nm}$ excitation. Fig. S36\$ displays the raw data and Fig. S39\$ the single wavelength kinetics and corresponding fits.

a concern is, however, the fact that the $\left(T_{1} T_{1}\right)_{\tau 1}$ spectra are very different for each dimer when using the parallel model. As such, the sequential model is more realistic for the global analysis. In this context, the transition between the different $\left(\mathrm{T}_{1} \mathrm{~T}_{1}\right)$ states does not necessarily correspond to an electronic transition, but is likely to relate to a relaxation process. As such, the two $\left(\mathrm{T}_{1} \mathrm{~T}_{1}\right)$ states in the kinetic model may correspond to the same electronic state. Once formed, the $\left(T_{1} T_{1}\right)_{\tau 2}$ state relaxes to the ground state. The underlying time constants for $\left(\mathrm{T}_{1} \mathrm{~T}_{1}\right)_{\tau 1}$ and $\left(\mathrm{T}_{1} \mathrm{~T}_{1}\right)_{\tau 2}$ are $269 \mathrm{ps} / 1.3 \mathrm{~ns}, 509 \mathrm{ps} / 1.3$ ns, and $173 \mathrm{ps} / 1.0 \mathrm{~ns}$ for $\mathbf{4 b}, \mathbf{4 c}$, and $\mathbf{4 d}$, respectively. For $\mathbf{4 a}$, only a single lifetime of $1.2 \mathrm{~ns}$ results. Notably, the pico- to nanosecond $\left(\mathrm{T}_{1} \mathrm{~T}_{1}\right)$ lifetimes in $\mathbf{4 a - d}$ are in stark contrast to the microsecond lifetimes of pentacene triplet excited states formed via intersystem crossing in, for example, TIBS. We take such a dramatic difference as support for the presence of two correlated local triplet excited states per dimer, that is, a correlated triplet $\left(\mathrm{T}_{1} \mathrm{~T}_{1}\right)$ pair. Here, fast deactivation via triplet-triplet annihilation occurs, as recently documented. ${ }^{80}$ As aforementioned, $\mathbf{4 a}\left(\mathrm{R}=\mathrm{SiMe}_{3}\right)$ gives rise to a single $\left(\mathrm{T}_{1} \mathrm{~T}_{1}\right)$ state at $80 \mathrm{~K}$ (mono-exponential decay), while for $\mathbf{4 b} \mathbf{b}-\mathbf{4 d}$ biexponential decays were found. As the substituent size increases from $\mathbf{4 a}$ to $\mathbf{4 b} \mathbf{b} \mathbf{- 4 d}$, it seems reasonable to postulate that the biphasic decay relates to geometric rearrangements of the substituents - this will be explained in more detail below.

\section{Transient absorption measurements in liquid solution at cryogenic temperature - a correlated triplet pair with excimer character}

Having established that singlet fission occurs in dimers 4a-d, we examine now the effect of intramolecular vibrations on the excited state dynamics. Therefore, fsTA measurements by using the same $730 \mathrm{~nm}$ excitation were performed at $157 \mathrm{~K}$ in liquid

MeTHF. Overall, a decrease of all excited state lifetimes is observed on moving from frozen to liquid solutions. This temperature dependence indicates activation barriers that may be related to geometric rearrangements - vide infra.

For $4 \mathrm{c}$ and $\mathrm{d}$, the $1400 \mathrm{~nm}$ fingerprint absorption of the pentacene singlet excited $\left(\mathrm{S}_{1} \mathrm{~S}_{0}\right)$ state is visible within the first 200 fs after excitation - Fig. S42.\$ In contrast, the acceleration of the singlet fission process for $\mathbf{4 a}$ and $\mathbf{b}$ leads to $\left(\mathrm{S}_{1} \mathrm{~S}_{0}\right)$ state lifetimes that are below our instrumental time resolution. Due to the short lifetime, it is only possible to deconvolute the singlet excited $\left(\mathrm{S}_{1} \mathrm{~S}_{0}\right)$ state by global analysis in the NIR region of $\mathbf{4 d}-$ Fig. S43.

Notably, the correlated triplet pair states $\left(\mathrm{T}_{1} \mathrm{~T}_{1}\right)$ of $\mathbf{4 a - c}$, which were at $80 \mathrm{~K}$ completely consistent with the sensitized triplet $\mathrm{T}_{1}$, appear much broader at $157 \mathrm{~K}-$ Fig. 8 and S41.t Considering this broadening in turn with the close proximity of the two triplet states, the two triplets of the correlated tripletpair are, in contrast to the $80 \mathrm{~K}$ experiments, electronically coupled and, in turn, (partially) delocalized at $157 \mathrm{~K}$. In detail, we hypothesize that such an electronic delocalization of the spin-correlated $\left(\mathrm{T}_{1} \mathrm{~T}_{1}\right)$ leads to a mixed state in the dimers. They feature a mix of delocalized excimer and localized triplet exited state character. In other words, the pure $\left(T_{1} T_{1}\right)$ states at $80 \mathrm{~K}$ possess a slight character of delocalization at $157 \mathrm{~K}$ and are denoted as "partially delocalized" $\left(T_{1} T_{1}\right)_{\tau 1}$ and $\left(T_{1} T_{1}\right)_{\tau 2}$.

Overall, the decay dynamics possess lifetimes for $\left(\mathrm{T}_{1} \mathrm{~T}_{1}\right)_{\tau 1}$ and $\left(\mathrm{T}_{1} \mathrm{~T}_{1}\right)_{\mathrm{\tau} 2}$ of 13 and $218 \mathrm{ps}$ for $\mathbf{4 a} ; 56$ and $318 \mathrm{ps}$ for $4 \mathbf{b} ; 21$ and $221 \mathrm{ps}$ for $4 \mathbf{c}$. For $4 \mathbf{d}$, the decay is tri-exponential with 4, 25, and 144 ps.

The $\left(\mathrm{T}_{1} \mathrm{~T}_{1}\right)$ decay dynamics, which are monoexponential for 4a and biexponential for $4 \mathbf{d}$ at $80 \mathrm{~K}$, are biexponential and even triexponential, respectively, at $157 \mathrm{~K}$. These observations are likely related to the size of the respective trialkylsilylsubstituents: 4a features the smallest substituents and $\mathbf{4 d}$ the largest. Importantly, the observed temperature-dependencies exclude the possibility of different triplet pair states per
A) EAS, $157 \mathrm{~K}, \mathrm{MeTHF}, 4 a$

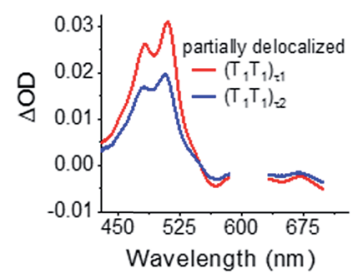

C) EAS, $157 \mathrm{~K}, \mathrm{MeTHF}, 4 \mathrm{c}$

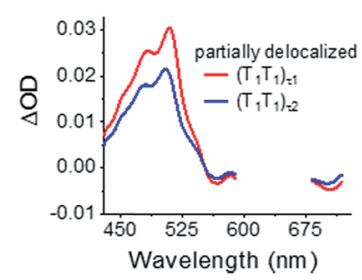

B) EAS, $157 \mathrm{~K}, \mathrm{MeTHF}$, 4b

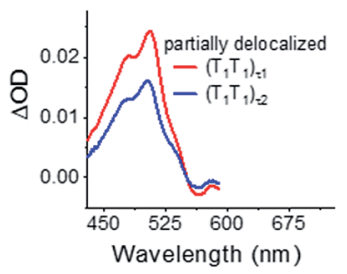

D) EAS, $157 \mathrm{~K}, \mathrm{MeTHF}, \mathbf{4 d}$

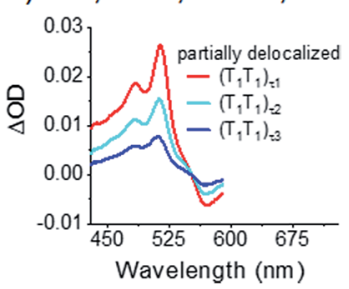

Fig. 8 EAS of dimers $4 a-d(A)-(D)$ measured in liquid argon-saturated MeTHF. The data were recorded at $157 \mathrm{~K}$. Spectra were obtained by global analysis with a sequential model of the transient absorption data measured upon femtosecond pump-probe experiments with $730 \mathrm{~nm}$ excitation. Fig. S41 displays the raw data and Fig. S44 the single wavelength kinetics and corresponding fits. 
molecule with different electronic natures. It is more likely that multiple conformations of the highly branched substituents exist in the excited state. Librations between these different conformations can lead to multi-exponential fits. ${ }^{81}$

\section{Transient absorption measurements at room temperature - pentacene excimer states}

As Fig. 5 indicates, the absorption features in the longwavelength region are weak at room temperature. Excitation into the lower Davydov bands at room temperature necessitated a change of solvent from MeTHF to benzonitrile - Fig. 3. Notable is, however, that such a solvent change and an increase in temperature are accompanied by a broadening and redshifting. Consequently, we used a $20 \mathrm{~nm}$ red-shifted excitation wavelength to excite exclusively into the lower Davydov band.

Again, a significant decrease of all excited state lifetimes is observed by increasing the temperatures from $157 \mathrm{~K}$ to room temperature. Upon photoexcitation at $750 \mathrm{~nm}$, typical fingerprint absorptions of the pentacene singlet excited $\left(\mathrm{S}_{1} \mathrm{~S}_{0}\right)$ state were not observed; most likely, due to a lifetime below our instrumental time resolution.

Compounds 4 a-c reveal two equally intense, broad and overlapping maxima in the visible range at around 480 and $510 \mathrm{~nm}$. These maxima are at the positions of the maxima of the correlated triplet pair states at $80 \mathrm{~K}$. The defined shape and intensity ratio are, however, completely lost upon going from 80 K to $295 \mathrm{~K}$ (see Fig. 10). Compound 4d shows a further feature at $440 \mathrm{~nm}$ - Fig. 9 and S45-S47. Additional minima in 4a-d at ca. 610-630 and 660-680 $\mathrm{nm}$ resemble the ground state absorption of the corresponding dimers. By virtue of the broad and featureless characteristics of the absorption bands at 480 and $510 \mathrm{~nm}$, which is very uncommon for pentacene excited states, we ascribe this feature to an excimer state.

It is, thus, hypothesized that the correlated triplet pair states become more and more delocalized as the temperature increases. This implies the two triplet excited states, one localized on each pentacene and electronically decoupled at $80 \mathrm{~K}$, transform with increasing temperature to an excimer state that is delocalized over both pentacenes of the dimer. To sum up these observations, we conclude that the states observed in room temperature experiments consist of two electronically interacting triplet excited states. These form a spin-correlated pair state $\left(\mathrm{T}_{1} \mathrm{~T}_{1}\right)$ over the entire temperature range. As the electronic interaction increases with temperature, the formation of a single excimer state is enabled, which is delocalized over the two pentacenes at room temperature.

Upon global analysis with a sequential model, two species, that is, $\left(\mathrm{T}_{1} \mathrm{~T}_{1}\right)$-excimer ${ }_{\tau 1}$ and $\left(\mathrm{T}_{1} \mathrm{~T}_{1}\right)$-excimer ${ }_{\tau 2}$, are identified for 4a-c - Fig. 9. Overall, the decay dynamics for the two excimers correlate with the size of the substituents: 5 and 50 ps for $4 \mathbf{4 a} ; 12$ and 85 ps for $4 \mathbf{b} ; 29$ and 99 ps for 4c. Again, the underlying substituent size-dependency corroborates our hypothesis that the multiexponential decays of the excimer states are caused by librations between different conformations of the substituents.

Compound $\mathbf{4 d}$ is a notable exception, and the decay is triexponential rather than biexponential with lifetimes of 2,11, and 76
A) EAS, $295 \mathrm{~K}, \mathrm{BN}, 4 \mathrm{a}$

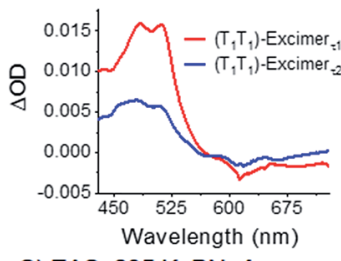

C) EAS, $295 \mathrm{~K}, \mathrm{BN}, 4 \mathrm{c}$

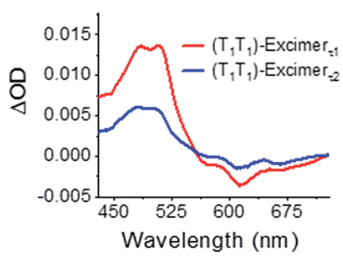

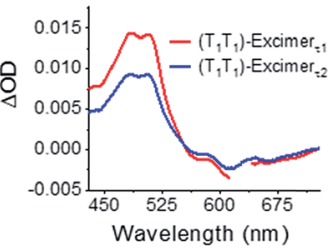

D) EAS, $295 \mathrm{~K}, \mathrm{BN}, \mathbf{4 d}$

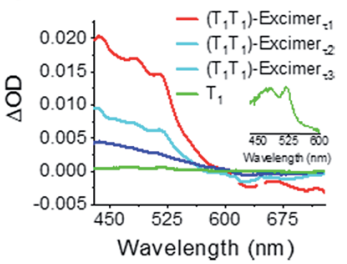

B) EAS, $295 \mathrm{~K}, \mathrm{BN}, 4 \mathrm{~b}$

Fig. 9 EAS of dimers $4 a-d(A)-(D)$ measured at room temperature in argon-saturated benzonitrile (BN). The data were recorded at room temperature ( $295 \mathrm{~K}$ ). Spectra were obtained by global analysis with a sequential model of the transient absorption data measured upon femtosecond pump-probe experiments with $750 \mathrm{~nm}$ excitation. Fig. S45\$ displays the raw data and Fig. S48\$ the single wavelength kinetics and corresponding fits.

ps that correspond to $\left(\mathrm{T}_{1} \mathrm{~T}_{1}\right)$-excimer ${ }_{\tau 1},\left(\mathrm{~T}_{1} \mathrm{~T}_{1}\right)$-excimer $\mathrm{ex2}_{\tau 2}$, and $\left(\mathrm{T}_{1} \mathrm{~T}_{1}\right)$-excimer $\mathrm{e}_{\tau 3}$, respectively. In addition, a small amount of a transient is identified with maxima at 480 and $520 \mathrm{~nm}$ which resembles the pentacene triplet excited state - Fig. 9D, inset. The lifetime exceeds several nanoseconds, although the amount formed is too low to determine the correct lifetime. We attribute this state to uncorrelated triplet $\left(\mathrm{T}_{1}+\mathrm{T}_{1}\right)$. The slightly different behavior of $\mathbf{4 d}$ versus the others of the series is rationalized by the notably increased electronic communication between the pentacenes and the pendent phenyl substituent of the alkyne.

\section{Summary of transient absorption measurements - the nature and role of the excimer states in singlet fission}

Upon photoexcitation of all four dimers $4 a-d$ at $80 \mathrm{~K}$, the signatures of a pentacene $\left(\mathrm{S}_{1} \mathrm{~S}_{0}\right)$ state are detectable and feature lifetimes close to the time resolution of our experimental setup (<200 fs). This singlet excited state transforms into a spincorrelated triplet pair state $\left(\mathrm{T}_{1} \mathrm{~T}_{1}\right)$, which mainly decays via triplet-triplet annihilation (TTA) back to the ground state (see Fig. 11). Moving to higher temperatures leads to an increase of molecular vibrational motion and to a scenario in which localization of the triplets on the individual pentacenes is lost (see Fig. 10). In other words, the two triplet excited states in $\left(\mathrm{T}_{1} \mathrm{~T}_{1}\right)$ are electronically coupled at room temperature by vibrational modes to form an excimer state. Extrapolation of the $\left(S_{1} S_{0}\right)$ lifetimes to room temperature implies that they are far shorter than the time resolution of our experimental setup, and, thus, not detectable. It is, therefore, impossible to draw a meaningful conclusion about the excimeric character of $\left(S_{1} S_{0}\right)$ at room temperature. Please note the work on microcrystalline pentacene films, which does show a Davydov splitting of the $\mathrm{S}_{1}$ $\leftarrow \mathrm{S}_{0}$ absorptions. ${ }^{65}$ Comparing $\mathbf{4 a}-\mathbf{d}$ with a recently published J-coupled pentacene dimer provides insight into the CTcharacter of $\left(S_{1} S_{0}\right)$. For example, a combination of excitonic 


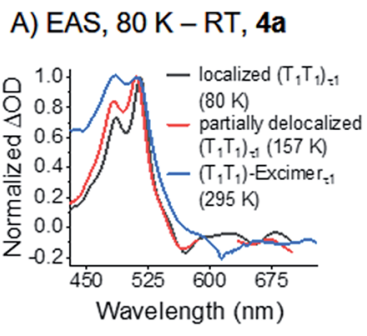

B) EAS, $80 \mathrm{~K}-\mathrm{RT}, \mathbf{4 b}$

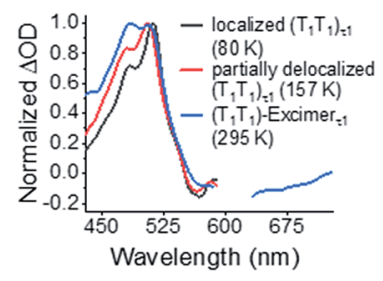

C) EAS, $80 \mathrm{~K}-\mathrm{RT}, 4 \mathrm{c}$

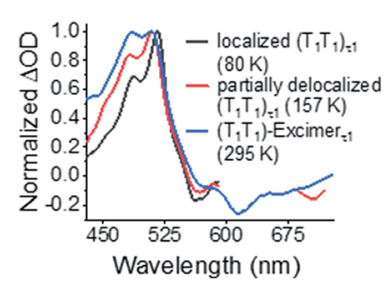

D) EAS, $80 \mathrm{~K}-\mathrm{RT}$, 4d

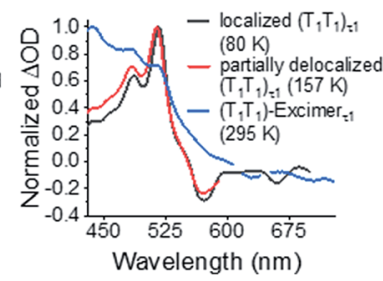

Fig. 10 EAS of the $\tau 1$ state of the correlated triplet pair of dimers $4 a-$ d (A)-(D) at different temperature. See Fig. 7-9 for the corresponding global fits.

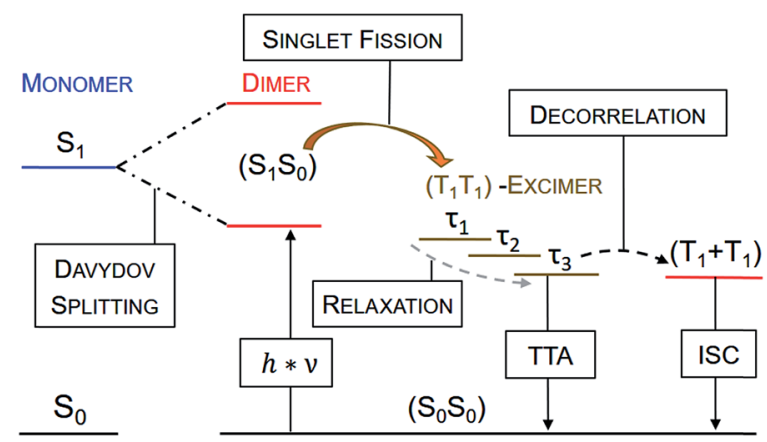

Fig. 11 Generalized mechanistic scheme illustrating all observed transitions and transient species at room temperature. No $\left(T_{1} T_{1}\right)$ excimer $_{\tau 3}$ was observed for dimers $4 a-c$. Triplet-triplet annihilation (TTA) is the main deactivation channel of the $\left(T_{1} T_{1}\right)$-excimer state for all dimers. Only dimer $4 d$ shows a minor amount of free triplet, which is most likely generated by decorrelation and, therefore, termed as $\left(T_{1}+\right.$ $\left.\mathrm{T}_{1}\right)$. The $\left(\mathrm{S}_{1} \mathrm{~S}_{0}\right)$ state is not directly observable at room temperature due to a too short lifetime, but its population is evidenced by measurements at cryogenic temperatures (see Fig. 6).

and CT-coupling in $\left(\mathrm{S}_{1} \mathrm{~S}_{0}\right)$ counteracts to the strong excitonic features in the ground-state absorption spectrum. ${ }^{43}$ As $4 a-$ d show a strong Davydov distortion in the ground-state, it is rather unlikely that $\left(\mathrm{S}_{1} \mathrm{~S}_{0}\right)$ of $\mathbf{4 a - d}$ mixes strongly with a CT-state. Moreover, a comparison of the $\left(\mathrm{T}_{1} \mathrm{~T}_{1}\right)$-excimers in $\mathbf{4 a}-\mathbf{d}$ with the absorption spectra of the TIPS-pentacene radical anion and radical cation did not reveal any CT-bands in the transient excimer spectra (Fig. S49 and S50 ).

\section{Conclusions}

A xanthene spacer has been used to link two pentacene chromophores and provides four dimers in which the pentacene moieties show direct intramolecular $\pi$-overlap in solution and the solid-state. These dimers have been designed specifically to explore processes that are linked to Davydov splitting and its effect on SF. To this end, quantum chemical calculations, steady state absorption, and electrochemical measurements have been used to corroborate that $\pi-\pi$-interactions cause Davydov bands. The absorption characteristics of the dimers suggest that the Davydov splitting depends on the solvent viscosity, and this premise is confirmed by temperature dependent absorption measurements in MeTHF between 80 and $295 \mathrm{~K}$. As such, the strength of the direct intramolecular $\pi$ overlap between the pentacene chromophores is dictated by vibrational motions. Calculations and observations both suggest that Davydov splitting is strengthened by mixing with other $\pi-\pi$ single excitations between unsymmetrical combinations of the $\pi$-orbitals of the individual pentacene moieties.

Transient absorption measurements establish the excited state dynamics of SF occurring after exciting the excitonically split states. Temperature-dependent assays in liquid and frozen solutions facilitate the observation, and subsequent identification of a correlated triplet $\left(\mathrm{T}_{1} \mathrm{~T}_{1}\right)$ pair at $80 \mathrm{~K}$, which becomes more and more coupled electronically upon increasing the temperature to form an excimer state at $295 \mathrm{~K}$ as the product of ultra-fast SF. Notably, the role and nature of the $\left(\mathrm{T}_{1} \mathrm{~T}_{1}\right)$-excimer states in $\mathbf{4 a - d}$ is not inconsistent with a recent study, in which an excimer state of likely $\left(\mathrm{T}_{1} \mathrm{~T}_{1}\right)$ character fails to promote triplet decorrelation: ${ }^{66}$ for tetracene monomers in solution, which lack fixed and/or defined orientation relative to each other, direct SF leading to decorrelated $T_{1}$ states occurs at intermolecular distances larger than active in excimer formation, where triplettriplet annihilation dominates. Note that SF is endothermic in tetracene but exothermic in pentacene. Additional differences exist: most importantly, $\mathbf{4 a - d}$ are rigid dimers with electronic ground-state interactions and directly accessible photoexcited dimer-states, as demonstrated by Davydov splitting. Nevertheless, the excimer states in the tetracene monomer solution and in 4a-d undergo both (mostly) triplet-triplet annihilation.

It would be very interesting to see if the tetracene excimer states can be localized by reducing the thermal energy. Such experiments are challenging, but the results would be of utmost importance for unraveling the true nature of the tetracene excimer. They would also provide further insight into the importance of photoexcitable dimer-states as a result of Davydov splitting.

Hopefully, the results presented will guide the way to such experiments and promote more facile analyses mechanisms of $\mathrm{SF}$ for acenes in a broad range of morphologies that might exhibit Davydov splitting such as, for example, aggregates, crystals, and films.

\section{Conflicts of interest}

There are no conflicts to declare.

\section{Acknowledgements}

The authors are grateful for support of their work in this area from the DFG Cluster of Excellence "Engineering of Advanced Materials” (grant numbers GU 517/17-1 and 182849149 - SFB 
953), "Solar Technologies go Hybrid" (an initiative of the Bavarian State Ministry for Science, Research and Art), and the Emerging Fields Initiative "Singlet Fission" (supported by FAU). In Canada support from the Natural Sciences and Engineering Research Council of Canada (NSERC) and the Canadian Foundation for Innovation (CFI) is gratefully acknowledged. B. S. B. gratefully acknowledges financial support in form of a $\mathrm{PhD}$ scholarship from "Studienstiftung des deutschen Volkes". We thank Dr Dan Lehnherr for the initial synthesis of compound S2. The authors acknowledge M. Thoss, P. B. Coto and R. R. Seelam for helpful discussions.

\section{Notes and references}

\begin{abstract}
\ Xanthene linked tetracenes have been reported. ${ }^{19,67}$
|| Compound 4d is unstable under voltage and its electrochemistry was, therefore, not further evaluated.

** The fingerprint at $450 \mathrm{~nm}$ is absent; probably due to the wavelength dependent character (chirp) of the instrument response function (IRF); it is with our setup stronger for measurements in the visible $(430-760 \mathrm{~nm})$ than in the NIR (800$1500 \mathrm{~nm})$. This renders an extraction of very short-lived species $(<200 \mathrm{fs})$ far more challenging for the visible than for the NIR.
\end{abstract}

1 J. C. Johnson, A. J. Nozik and J. Michl, Acc. Chem. Res., 2013, 46, 1290-1299.

2 M. B. Smith and J. Michl, Annu. Rev. Phys. Chem., 2013, 64, 361-386.

3 M. B. Smith and J. Michl, Chem. Rev., 2010, 110, 6891-6936.

4 A. Rao and R. H. Friend, Nat. Rev. Mater., 2017, 2, 17063.

5 D. Casanova, Chem. Rev., 2018, 118, 7164-7207.

6 S. Singh, W. J. Jones, W. Siebrand, B. P. Stoicheff and W. G. Schneider, J. Chem. Phys., 1965, 42, 330-342.

7 C. E. Swenberg and W. T. Stacy, Chem. Phys. Lett., 1968, 2, 327-328.

8 N. Geacintov, M. Pope and F. Vogel, Phys. Rev. Lett., 1969, 22, 593-596.

9 W. Shockley and H. J. Queisser, J. Appl. Phys., 1961, 32, 510519.

10 D. N. Congreve, J. Lee, N. J. Thompson, E. Hontz, S. R. Yost, P. D. Reusswig, M. E. Bahlke, S. Reineke, T. Van Voorhis and M. A. Baldo, Science, 2013, 340, 334-337.

11 L. Yang, M. Tabachnyk, S. L. Bayliss, M. L. Böhm, K. Broch, N. C. Greenham, R. H. Friend and B. Ehrler, Nano Lett., 2015, 15, 354-358.

12 S. R. Yost, J. Lee, M. W. B. Wilson, T. Wu, D. P. McMahon, R. R. Parkhurst, N. J. Thompson, D. N. Congreve, A. Rao, K. Johnson, M. Y. Sfeir, M. G. Bawendi, T. M. Swager, R. H. Friend, M. A. Baldo and T. Van Voorhis, Nat. Chem., 2014, 6, 492-497.

13 B. Ehrler, K. P. Musselman, M. L. Böhm, R. H. Friend and N. C. Greenham, Appl. Phys. Lett., 2012, 101, 153507.

14 P. J. Jadhav, P. R. Brown, N. Thompson, B. Wunsch, A. Mohanty, S. R. Yost, E. Hontz, T. Van Voorhis, M. G. Bawendi, V. Bulović and M. A. Baldo, Adv. Mater., 2012, 24, 6169-6174.

15 T. C. Berkelbach, M. S. Hybertsen and D. R. Reichman, J. Chem. Phys., 2013, 138, 114102.
16 G. B. Piland, J. J. Burdett, R. J. Dillon and C. J. Bardeen, J. Phys. Chem. Lett., 2014, 5, 2312-2319.

17 W. L. Chan, M. Ligges, A. Jailaubekov, L. Kaake, L. MiajaAvila and X. Y. Zhu, Science, 2011, 334, 1541-1545.

18 S. T. Roberts, R. E. McAnally, J. N. Mastron, D. H. Webber, M. T. Whited, R. L. Brutchey, M. E. Thompson and S. E. Bradforth, J. Am. Chem. Soc., 2012, 134, 6388-6400.

19 N. V. Korovina, S. Das, Z. Nett, X. Feng, J. Joy, R. Haiges, A. I. Krylov, S. E. Bradforth and M. E. Thompson, J. Am. Chem. Soc., 2016, 138, 617-627.

20 T. Yamakado, S. Takahashi, K. Watanabe, Y. Matsumoto, A. Osuka and S. Saito, Angew. Chem., 2018, 130, 5536-5541.

21 N. R. Monahan, D. Sun, H. Tamura, K. W. Williams, B. Xu, Y. Zhong, B. Kumar, C. Nuckolls, A. R. Harutyunyan, G. Chen, H.-L. Dai, D. Beljonne, Y. Rao and X. Y. Zhu, Nat. Chem., 2017, 9, 341-346.

22 H. L. Stern, A. Cheminal, S. R. Yost, K. Broch, S. L. Bayliss, K. Chen, M. Tabachnyk, K. Thorley, N. Greenham, J. M. Hodgkiss, J. Anthony, M. Head-Gordon, A. J. Musser, A. Rao and R. H. Friend, Nat. Chem., 2017, 9, 1205.

23 J. C. Johnson, A. J. Nozik and J. Michl, J. Am. Chem. Soc., 2010, 132, 16302-16303.

24 J. N. Schrauben, J. L. Ryerson, J. Michl and J. C. Johnson, J. Am. Chem. Soc., 2014, 136, 7363-7373.

25 A. Akdag, A. Wahab, P. Beran, L. Rulíšek, P. I. Dron, J. Ludvík and J. Michl, J. Org. Chem., 2015, 80, 80-89.

26 J. Yu, L.-M. Fu, L.-J. Yu, Y. Shi, P. Wang, Z.-Y. Wang-Otomo and J.-P. Zhang, J. Am. Chem. Soc., 2017, 139, 15984-15993.

27 A. J. Musser, M. Maiuri, D. Brida, G. Cerullo, R. H. Friend and J. Clark, J. Am. Chem. Soc., 2015, 137, 5130-5139.

28 S. W. Eaton, L. E. Shoer, S. D. Karlen, S. M. Dyar, E. A. Margulies, B. S. Veldkamp, C. Ramanan, D. A. Hartzler, S. Savikhin, T. J. Marks and M. R. Wasielewski, J. Am. Chem. Soc., 2013, 135, 1470114712 .

29 E. A. Margulies, C. E. Miller, Y. Wu, L. Ma, G. C. Schatz, R. M. Young and M. R. Wasielewski, Nat. Chem., 2016, 8, 1120.

30 E. A. Margulies, J. L. Logsdon, C. E. Miller, L. Ma, E. Simonoff, R. M. Young, G. C. Schatz and M. R. Wasielewski, J. Am. Chem. Soc., 2017, 139, 663-671.

31 P. E. Hartnett, E. A. Margulies, C. M. Mauck, S. A. Miller, Y. Wu, Y.-L. Wu, T. J. Marks and M. R. Wasielewski, J. Phys. Chem. B, 2016, 120, 1357-1366.

32 C. E. Miller, M. R. Wasielewski and G. C. Schatz, J. Phys. Chem. C, 2017, 121, 10345-10350.

33 S. Lukman, J. M. Richter, L. Yang, P. Hu, J. Wu, N. C. Greenham and A. J. Musser, J. Am. Chem. Soc., 2017, 139, 18376-18385.

34 J. J. Burdett and C. J. Bardeen, J. Am. Chem. Soc., 2012, 134, 8597-8607.

35 E. G. Fuemmeler, S. N. Sanders, A. B. Pun, E. Kumarasamy, T. Zeng, K. Miyata, M. L. Steigerwald, X. Y. Zhu, M. Y. Sfeir, L. M. Campos and N. Ananth, ACS Cent. Sci., 2016, 2, 316324.

36 B. S. Basel, J. Zirzlmeier, C. Hetzer, S. R. Reddy, B. T. Phelan, M. D. Krzyaniak, P. B. Coto, M. K. Volland, R. M. Young, 
T. Clark, M. Thoss, R. R. Tykwinski, M. R. Wasielewski and D. M. Guldi, Chem, 2018, 4, 1092-1111.

37 T. C. Berkelbach, M. S. Hybertsen and D. R. Reichman, J. Chem. Phys., 2013, 138, 114103.

38 M. H. Farag and A. I. Krylov, J. Phys. Chem. C, 2018, 122, 25753-25763.

39 M. Chen, Y. J. Bae, C. M. Mauck, A. Mandal, R. M. Young and M. R. Wasielewski, J. Am. Chem. Soc., 2018, 140, 9184-9192.

40 B. S. Basel, J. Zirzlmeier, C. Hetzer, B. T. Phelan, M. D. Krzyaniak, S. R. Reddy, P. B. Coto, N. E. Horwitz, R. M. Young, F. J. White, F. Hampel, T. Clark, M. Thoss, R. R. Tykwinski, M. R. Wasielewski and D. M. Guldi, Nat. Commun., 2017, 8, 15171.

41 E. Kumarasamy, S. N. Sanders, M. J. Y. Tayebjee, A. Asadpoordarvish, T. J. H. Hele, E. G. Fuemmeler, A. B. Pun, L. M. Yablon, J. Z. Low, D. W. Paley, J. C. Dean, B. Choi, G. D. Scholes, M. L. Steigerwald, N. Ananth, D. R. McCamey, M. Y. Sfeir and L. M. Campos, J. Am. Chem. Soc., 2017, 139, 12488-12494.

42 T. Sakuma, H. Sakai, Y. Araki, T. Mori, T. Wada, N. V. Tkachenko and T. Hasobe, J. Phys. Chem. A, 2016, 120, 1867-1875.

43 S. Lukman, K. Chen, J. M. Hodgkiss, D. H. P. Turban, N. D. M. Hine, S. Dong, J. Wu, N. C. Greenham and A. J. Musser, Nat. Commun., 2016, 7, 13622.

44 M. J. Y. Tayebjee, S. N. Sanders, E. Kumarasamy, L. M. Campos, M. Y. Sfeir and D. R. McCamey, Nat. Phys., 2017, 13, 182-188.

45 S. Lukman, A. J. Musser, K. Chen, S. Athanasopoulos, C. K. Yong, Z. Zeng, Q. Ye, C. Chi, J. M. Hodgkiss, J. Wu, R. H. Friend and N. C. Greenham, Adv. Funct. Mater., 2015, 25, 5452-5461.

46 H. Yamagata, J. Norton, E. Hontz, Y. Olivier, D. Beljonne, J. L. Brédas, R. J. Silbey and F. C. Spano, J. Chem. Phys., 2011, 134, 204703.

47 J. E. Anthony, Chem. Rev., 2006, 106, 5028-5048.

48 J. E. Anthony, Angew. Chem., Int. Ed., 2008, 47, 452-483.

49 Q. Miao, X. Chi, S. Xiao, R. Zeis, M. Lefenfeld, T. Siegrist, M. L. Steigerwald and C. Nuckolls, J. Am. Chem. Soc., 2006, 128, 1340-1345.

50 E. Busby, T. C. Berkelbach, B. Kumar, A. Chernikov, Y. Zhong, H. Hlaing, X. Y. Zhu, T. F. Heinz, M. S. Hybertsen, M. Y. Sfeir, D. R. Reichman, C. Nuckolls and O. Yaffe, J. Am. Chem. Soc., 2014, 136, 10654-10660.

51 S. Tretiak, W. M. Zhang, V. Chernyak and S. Mukamel, Proc. Natl. Acad. Sci. U. S. A., 1999, 96, 13003.

52 J. D. Cook, T. J. Carey and N. H. Damrauer, J. Phys. Chem. A, 2016, 120, 4473-4481.

53 T. J. Carey, J. L. Snyder, E. G. Miller, T. Sammakia and N. H. Damrauer, J. Org. Chem., 2017, 82, 4866-4874.

54 X. Feng and A. I. Krylov, Phys. Chem. Chem. Phys., 2016, 18, 7751-7761.

55 J. Seibt, P. Marquetand, V. Engel, Z. Chen, V. Dehm and F. Würthner, Chem. Phys., 2006, 328, 354-362.

56 M. J. Y. Tayebjee, R. G. C. R. Clady and T. W. Schmidt, Phys. Chem. Chem. Phys., 2013, 15, 14797-14805.
57 S.-H. Lim, T. G. Bjorklund, F. C. Spano and C. J. Bardeen, Phys. Rev. Lett., 2004, 92, 107402.

58 M. J. Y. Tayebjee, K. N. Schwarz, R. W. MacQueen, M. Dvořák, A. W. C. Lam, K. P. Ghiggino, D. R. McCamey, T. W. Schmidt and G. J. Conibeer, J. Phys. Chem. C, 2016, 120, 157-165.

59 D. Beljonne, H. Yamagata, J. L. Brédas, F. C. Spano and Y. Olivier, Phys. Rev. Lett., 2013, 110, 226402.

60 K. Kolata, T. Breuer, G. Witte and S. Chatterjee, ACS Nano, 2014, 8, 7377-7383.

61 C. M. Mauck, P. E. Hartnett, E. A. Margulies, L. Ma, C. E. Miller, G. C. Schatz, T. J. Marks and M. R. Wasielewski, J. Am. Chem. Soc., 2016, 138, 11749-11761.

62 K. E. Brown, W. A. Salamant, L. E. Shoer, R. M. Young and M. R. Wasielewski, J. Phys. Chem. Lett., 2014, 5, 2588-2593.

63 K. M. Lefler, K. E. Brown, W. A. Salamant, S. M. Dyar, K. E. Knowles and M. R. Wasielewski, J. Phys. Chem. A, 2013, 117, 10333-10345.

64 H. L. Stern, A. J. Musser, S. Gelinas, P. Parkinson, L. M. Herz, M. J. Bruzek, J. Anthony, R. H. Friend and B. J. Walker, Proc. Natl. Acad. Sci. U. S. A., 2015, 112, 7656-7661.

65 H. Marciniak, I. Pugliesi, B. Nickel and S. Lochbrunner, Phys. Rev. B: Condens. Matter Mater. Phys., 2009, 79, 235318.

66 C. B. Dover, J. K. Gallaher, L. Frazer, P. C. Tapping, A. J. Petty Ii, M. J. Crossley, J. E. Anthony, T. W. Kee and T. W. Schmidt, Nat. Chem., 2018, 10, 305.

67 H. Liu, V. M. Nichols, L. Shen, S. Jahansouz, Y. Chen, K. M. Hanson, C. J. Bardeen and X. Li, Phys. Chem. Chem. Phys., 2015, 17, 6523-6531.

68 S. R. Reddy, P. B. Coto and M. Thoss, Chem. Phys., 2018, 515, 628-634.

69 D. Lehnherr, A. H. Murray, R. McDonald and R. R. Tykwinski, Angew. Chem., Int. Ed., 2010, 49, 6190-6194.

70 Y. Morisaki and Y. Chujo, Tetrahedron Lett., 2005, 46, 25332537.

71 J. L. Marshall, D. Lehnherr, B. D. Lindner and R. R. Tykwinski, ChemPlusChem, 2017, 82, 967-1001.

72 A. D. Becke, J. Chem. Phys., 1993, 98, 5648-5652.

73 J.-D. Chai and M. Head-Gordon, Phys. Chem. Chem. Phys., 2008, 10, 6615-6620.

74 S. Grimme, J. Antony, S. Ehrlich and H. Krieg, J. Chem. Phys., 2010, 132, 154104.

75 R. Ditchfield, W. J. Hehre and J. A. Pople, J. Chem. Phys., 1971, 54, 724-728.

76 M. J. S. Dewar, E. G. Zoebisch, E. F. Healy and J. J. P. Stewart, J. Am. Chem. Soc., 1985, 107, 3902-3909.

77 F. Würthner, C. R. Saha-Möller, B. Fimmel, S. Ogi, P. Leowanawat and D. Schmidt, Chem. Rev., 2016, 116, 962-1052.

78 F. C. Spano, Annu. Rev. Phys. Chem., 2006, 57, 217-243.

79 CRC Handbook of Chemistry and Physics, ed. J. R. Rumble, CRC Press, Taylor \& Francis, 2018.

80 J. Zirzlmeier, D. Lehnherr, P. B. Coto, E. T. Chernick, R. Casillas, B. S. Basel, M. Thoss, R. R. Tykwinski and D. M. Guldi, Proc. Natl. Acad. Sci. U. S. A., 2015, 112, 5325-5330.

81 F. R. Beierlein, O. G. Othersen, H. Lanig, S. Schneider and T. Clark, J. Am. Chem. Soc., 2006, 128, 5142-5152. 